\title{
Évaluer l'efficacité de l'éducation relative au changement climatique en milieu non formel : une étude de cas
}

Valériane Champagne St-Arnaud et Ghislain Samson

\section{CpenEdition}

Journals

Édition électronique

URL : https://journals.openedition.org/ere/8097

ISSN : 2561-2271

Éditeur

Centr'ERE

\section{Référence électronique}

Valériane Champagne St-Arnaud et Ghislain Samson, «Évaluer l'efficacité de l'éducation relative au changement climatique en milieu non formel : une étude de cas ", Éducation relative à l'environnement [En ligne], Volume 17-1 | 2022, mis en ligne le 27 janvier 2022, consulté le 02 février 2022. URL : http:// journals.openedition.org/ere/8097

Ce document a été généré automatiquement le 2 février 2022

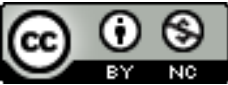

La revue Éducation relative à l'environnement est mise à disposition selon les termes de la Licence Creative Commons Attribution - Pas d'Utilisation Commerciale 4.0 International. 


\title{
Évaluer l'efficacité de l'éducation relative au changement climatique en milieu non formel : une étude de
}

\section{cas}

\author{
Valériane Champagne St-Arnaud et Ghislain Samson
}

1 Pour stimuler l'action environnementale chez les jeunes, de plus en plus de praticiens délaissent les traditionnels programmes éducatifs - visant à transmettre des connaissances, souvent dans un contexte formel - pour adopter une approche plus holistique et participative, de nature à développer un ensemble de compétences, la pensée critique et l'implication citoyenne (Bonnett, 2002; Arnold et coll., 2009). Or, selon plusieurs chercheurs, démontrer l'efficacité de ces activités non formelles est une tâche complexe. Contrairement aux programmes d'éducation en milieu formel, l'évaluation des activités de mobilisation jeunesse en environnement n'a pas fait l'objet de nombreuses études, comme le soulignent Riemer et coll. (2014). Dans ce contexte, proposer un modèle d'évaluation s'appuyant sur un socle théorique multidisciplinaire semble une avenue prometteuse, à la fois du point de vue de la recherche académique que de la pratique sur le terrain.

\section{Problématique}

2 Selon les données du plus récent Baromètre de l'action climatique au Québec, près de $96 \%$ de la population québécoise croit que le changement climatique concerne leur génération et les trois quarts estiment qu'il est urgent d'agir contre ce phénomène (Champagne St-Arnaud et Daignault, 2020). Cette prise de conscience intergénérationnelle constitue une étape importante vers l'adoption de mesures individuelles et collectives pour atténuer la crise environnementale la plus importante $\mathrm{du} 21^{\mathrm{e}}$ siècle (Groupe d'experts intergouvernemental sur l'évolution du climat [GIEC], 
2018). La technologie étant insuffisante à elle seule pour résoudre cette problématique, la mobilisation de toutes les personnes est essentielle (Stocknes, 2015).

3 Toutefois, plusieurs chercheurs.euses soulignent la pertinence de diriger les efforts de mobilisation environnementale auprès des adolescent.e.s, et ce, pour trois raisons. D'une part, bien que les conséquences du changement climatique se manifestent à un rythme de plus en plus rapide (GIEC, 2018), la jeune génération sera la plus susceptible d'en subir les effets au cours des prochaines décennies (Ojala, 2012). D'autre part, c'est généralement à l'adolescence qu'un individu forge son identité. Si un.e jeune est amené.e à se percevoir dès maintenant comme une personne préoccupée par les questions environnementales, cela favorisera l'enracinement et le maintien de cette perception dans le futur (Arnett, 2006; Blythe et Harré, 2012). Finalement, l'histoire montre que les jeunes ont souvent été aux premières loges - et continuent de l'être - de mouvements visant des changements de paradigmes à l'échelle sociétale (Ballantyne et coll., 1998; Ginwright et James, 2002). Greta Thunberg - qui a mobilisé des milliers d'étudiant.e.s à travers le monde dans des "grèves pour le climat» - en constitue un exemple récent. Selon Riemer et coll. (2014), cela confère à la jeune génération " [une place déterminante] dans toute tentative visant à solutionner des problématiques environnementales par le biais du changement social » (p. 555, traduction libre).

\section{Les activités d'éducation relative à l'environnement et au changement climatique auprès des jeunes}

En cohérence avec les constats précédents, de nombreuses activités éducatives autour de la question du changement climatique ont été déployées auprès des jeunes, la plupart en milieu formel. Toutefois, plusieurs chercheurs.euses soulignent l'importance de développer parallèlement des interventions éducatives en milieu non formel, par exemple, dans des camps de jour et des musées. (Bonnett, 2002 ; Davis, 2010 ; Riemer et coll., 2014 ; Champagne-Poirier et coll., 2017). À leur avis, le milieu non formel constitue un terreau favorable à une approche holistique permettant non seulement l'acquisition de connaissances environnementales (Simard et Samson, 2018; Simard et Samson, soumis), mais également le développement de compétences essentielles à l'engagement social, comme la pensée critique, le leadership, l'empathie (Jensen et Schnack, 1997; Jensen, 2002 ; Schusler et coll., 2009). Selon Sauvé (2013), ces compétences éthiques, critiques, politiques, associées aux compétences scientifiques, contribuent au développement d'une écocitoyenneté, soit "une citoyenneté consciente des liens étroits entre société et nature, une citoyenneté critique, compétente, créative et engagée, capable et désireuse de participer aux débats publics, à la recherche de solutions et à l'innovation écosociale » (p. 2-3).

5 Ainsi, l'écocitoyenneté dépasse la simple adoption d'écogestes à l'échelle individuelle (comme recycler ou prendre l'autobus) et s'incarne également dans l'action environnementale à l'échelle sociale, que Alisat et Riemer (2015) décrivent comme « un comportement civique adopté consciemment et intentionnellement dans l'objectif de créer un effet positif (souvent indirect) sur l'environnement par le biais du changement collectif et, à divers degrés, politique » (p. 14, traduction libre). L'adoption d'écogestes est néanmoins souvent au cœur des activités d'éducation relative à l'environnement et au changement climatique (ERCC), dans une perspective gradualiste conduisant progressivement à un engagement social plus marqué. 


\section{L'évaluation de l'efficacité des activités d'éducation relative à l'environnement et au changement climatique en milieu non formel}

6 Dans la documentation scientifique consultée, un consensus émerge quant à l'importance d'évaluer l'efficacité des activités d'ERCC à partir d'un cadre théorique bien défini (Riemer et coll., 2014). Or, évaluer l'efficacité de telles activités est complexe pour deux raisons. D'une part, alors qu'il existe une importante littérature portant sur l'évaluation de l'ERCC en milieu formel, celle qui concerne le milieu non formel est beaucoup moins abondante, particulièrement à l'égard des activités destinées aux jeunes (Guevara et coll., 2009; Riemer et coll., 2014). D'autre part, il s'agit principalement d'études empiriques exposant différents effets de la participation de jeunes à des activités d'ERCC, mais qui ne s'appuient pas nécessairement sur un cadre théorique global.

7 Certain.e.s chercheurs.euses ont utilisé des instruments permettant de mesurer l'adoption effective de comportements écoresponsables au niveau civique, notamment l'échelle de l'action environnementale (Alisat et Riemer, 2015 ; Riemer et coll., 2016). Ce type de mesure présente toutefois une lacune importante, car elle évalue le résultat et non le processus. Or il importe de rendre compte du fait que, même si les jeunes n'ont pas posé de gestes concrets à l'issue de leur participation à une activité d'ERCC, ils ont toutefois cheminé personnellement en développant de nouvelles connaissances ou de nouvelles attitudes favorables à l'adoption éventuelle de comportements écocitoyens.

D'autres études, comme celles de Trott et coll. (2020), Dittmer et coll. (2018) ou de Kerry et coll. (2014), ont mesuré l'impact d'activités d'ERCC sur des variables bien précises du processus de changement (par exemple, le sentiment d'auto-efficacité ou l'espoir), mais sans les examiner dans un cadre plus global.

9 En somme, à notre connaissance, aucune étude portant sur l'évaluation de l'efficacité d'une activité d'ERCC en milieu non formel ne s'est explicitement inspirée d'un modèle théorique pour évaluer l'ensemble du processus d'adoption d'un comportement écoresponsable individuel ou social chez de jeunes participant.e.s.

Dans ce contexte, cet article a une double visée: (1) proposer un cadre d'évaluation d'une activité d'ERCC destinée aux jeunes, à partir du modèle théorique de Klöckner (2015) et (2) l'appliquer à une étude de cas en milieu non formel, soit l'édition 2019 de la campagne Sors de ta bulle mise en œuvre par la Fondation Monique-Fitz-Back et dont l'objectif était de mobiliser les jeunes de 12 à 17 ans dans la lutte climatique.

\section{Cadre théorique}

11 La littérature des champs de l'éducation relative à l'environnement, de la psychologie environnementale et du marketing social permet de constater que de nombreux chercheurs.euses se sont intéressé.e.s aux facteurs expliquant l'adoption (ou non) de comportements écoresponsables. Par exemple, la méta-analyse de Bamberg et Möser (2007) a identifié sept déterminants psychosociaux de l'intention d'adopter un tel comportement: les attitudes, le contrôle comportemental perçu, les normes morales personnelles, la prise de conscience du problème, les normes sociales, le sentiment de culpabilité et le processus d'attribution. D'autres facteurs externes ou internes (par 
exemple, le contexte ou les habitudes) peuvent également favoriser ou freiner le comportement écoresponsable (Steg et Vlek, 2009). Plusieurs modèles théoriques ont été élaborés pour exposer le rôle de ces facteurs dans le processus de changement comportemental, dont l'approche de l'action raisonnée (Fishbein et Ajzen, 2010), le modèle valeurs-croyances-normes (Stern, 2000), le modèle du changement de comportement autorégulé (Bamberg, 2013) et le modèle global de la détermination de l'action (Klöckner, 2015).

Parmi ces modèles théoriques exposant les variables impliquées dans le processus d'adoption d'un comportement écoresponsable, le modèle global de la détermination de l'action (Klöckner, 2015) (figure 1) apparaît particulièrement pertinent pour évaluer l'efficacité d'une activité d'ERCC, et ce, pour deux raisons. D'une part, il constitue l'un des modèles les plus exhaustifs puisqu'il combine les variables de modèles précédents (l'approche de l'action raisonnée et le modèle valeurs-croyances-normes), en plus d'y ajouter des variables importantes comme les contraintes objectives et les habitudes, le tout dans une structure logique qui traduit l'enchainement des étapes de changement. D'autre part, ce modèle a été validé empiriquement par de nombreuses études (Sopha et Klöckner, 2011 ; Klöckner et Oppedal, 2011 ; van den Broek et coll., 2019 ; Joanes et coll., 2020).

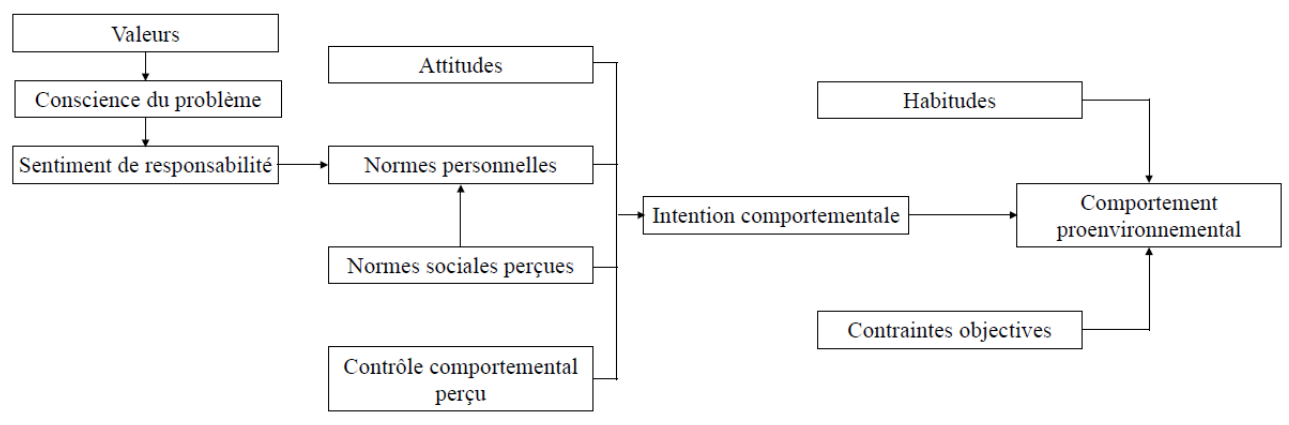

Figure1. Modèle global de la détermination de l'action (traduction libre et adaptation de Klöckner, 2015, p. 92)

\section{Méthodologie}

13 Puisque le premier objectif de la recherche que nous avons menée est de proposer un cadre d'évaluation d'une activité d'ERCC destinée aux jeunes, il convient d'expliciter en premier lieu, la façon dont le modèle de Klöckner (2015) a été traduit en indicateurs d'efficacité. Nous exposons ensuite la façon dont ces indicateurs ont été utilisés dans le contexte de l'étude de cas de la campagne Sors de ta bulle, correspondant au second objectif de cet article. La posture épistémologique ainsi que le devis ayant guidé cette étude de cas y sont brièvement exposés et justifiés.

\section{Élaboration du cadre d'évaluation}

À partir du modèle global de la détermination de l'action (Klöckner, 2015), il a été possible d'élaborer une liste de douze indicateurs de l'efficacité d'une activité d'ERCC, regroupés autour de trois dimensions du comportement écoresponsable : la motivation, le sentiment d'efficacité et le passage à l'action (tableau 1). 
Des exemples d'énoncés démontrent de quelle façon les indicateurs peuvent être intégrés concrètement à un outil de mesure. Une attention particulière a été apportée à formuler des énoncés et des choix de réponses permettant de mesurer la plus grande diversité de positions possible. Cela apparait important dans la mesure où le plus récent Baromètre de l'action climatique (Champagne St-Arnaud et coll., 2020) a révélé que la population québécoise se divise en six groupes distincts au regard de leurs attitudes et comportements relatifs à l'action climatique. Certains groupes se démarquent notamment par leur indifférence à la crise climatique et leur perception qu'il n'est pas nécessaire de s'investir dans cette lutte. D'autres études similaires - par exemple, celles menées annuellement par le Yale Program on Climate Change Communication - confirment la présence de segments réfractaires à la lutte climatique au sein d'autres populations ailleurs dans le monde (Goldberg et coll., 2020).

Tableau 1. Indicateurs d'efficacité d'une activité d'ERECC spécifique à l'enjeu du changement climatique

\begin{tabular}{|c|c|c|}
\hline Dimensions & Indicateurs & Exemples d'énoncés \\
\hline \multirow[t]{6}{*}{ Motivation } & $\begin{array}{l}\text { Niveau de connaissances perçu } \\
\text { des causes et des conséquences } \\
\text { du changement climatique }\end{array}$ & $\begin{array}{l}\text { "Le changement climatique est un sujet dont } \\
\text { je comprends [très bien, assez bien, peu, pas } \\
\text { du tout] les causes et les conséquences. " }\end{array}$ \\
\hline & $\begin{array}{l}\text { Niveau de motivation générale à } \\
\text { s'impliquer sur le plan citoyen }\end{array}$ & $\begin{array}{l}\text { "De façon gènérale, jouer un rôle actif dans } \\
\text { la société est pour moi [tress, assez, peu, pas } \\
\text { du tout] important. " }\end{array}$ \\
\hline & $\begin{array}{l}\text { Niveau de motivation spécifique } \\
\text { à faire sa part dans la lutte } \\
\text { climatique }\end{array}$ & $\begin{array}{l}\text { "De façon spécifique, m' impliquer dans la } \\
\text { lutte climatique est pour moi [tres, assez, peu, } \\
\text { pas du tout] important. }\end{array}$ \\
\hline & $\begin{array}{l}\text { Attitudes relatives à la lutte } \\
\text { climatique }\end{array}$ & $\begin{array}{l}\text { "S Selon moi, les actions individuelles sont } \\
\text { [très, plutôt peu, pas du tout] utiles pour lutter } \\
\text { contre le changement climatique. " }\end{array}$ \\
\hline & $\begin{array}{l}\text { Normes descriptives perçues } \\
\text { relatives à la lutte climatique }\end{array}$ & $\begin{array}{l}\text { Niveau d'accord avec l'énoncé suivant : «De } \\
\text { plus en plus de jeunes s' impliquent dans la } \\
\text { luutte climatique. . }\end{array}$ \\
\hline & $\begin{array}{l}\text { Normes prescriptives perçues } \\
\text { relatives à ta lutte climatique }\end{array}$ & $\begin{array}{l}\text { Niveau d'accord avec l'énoncé suivant : } \\
\text { " M M impliquer dans la lutte climatique est vu } \\
\text { de mantère positive par mes amis, mes } \\
\text { parents, mes enseignants, etc. }\end{array}$ \\
\hline \multirow[t]{3}{*}{$\begin{array}{l}\text { Sentiment } \\
\text { d'efficacité }\end{array}$} & $\begin{array}{l}\text { Contrôle personnel perçu relatif } \\
\text { à la lutte climatique }\end{array}$ & $\begin{array}{l}\text { Niveau d'accord avec l'énoncé suivant: \& Je } \\
\text { sais quoi faire pour diminuer personnellement } \\
\text { mon impact climatique..) }\end{array}$ \\
\hline & $\begin{array}{l}\text { Habiletés perçues relatives à la } \\
\text { lutte climatique }\end{array}$ & $\begin{array}{l}\text { Niveau d'accord avec l'énoncé suivant : \& Je } \\
\text { suis capable de convaincre mon entourage de } \\
\text { diminuer son impact climatique. }\end{array}$ \\
\hline & $\begin{array}{l}\text { Contrôle collectif perçu relatif à } \\
\text { la lutte climatique }\end{array}$ & $\begin{array}{l}\text { Niveau d'accord avec l'énoncé suivant : « Si } \\
\text { les jeunes du Québec se mettent en } \\
\text { movvenent, on peut faire une différence dans } \\
\text { la lutte climatique. }\end{array}$ \\
\hline \multirow[t]{3}{*}{$\begin{array}{l}\begin{array}{l}\text { Passage à } \\
\text { l'action }\end{array} \\
\end{array}$} & $\begin{array}{l}\text { Niveau de l'intention de } \\
\text { s'impliquer concrètement dans } \\
\text { la lutte climatique }\end{array}$ & $\begin{array}{l}\text { Niveau d'accord avec l'énoncé suivant : « J'ai } \\
\text { l'intention de m' impliquer dans un projet de } \\
\text { lutte climatique. }\end{array}$ \\
\hline & $\begin{array}{l}\text { Niveau perçu de l'intensité de } \\
\text { l'implication dans la lutte } \\
\text { climatique }\end{array}$ & $\begin{array}{l}\text { « Dans ma vie quotidienne (ex. choix de } \\
\text { transport, choix alimentaires, etc.), je me } \\
\text { considere actuellement [tres, assez, peu, pas } \\
\text { du toutl] actif ou active en matiere de lutte } \\
\text { climatique. 》 }\end{array}$ \\
\hline & $\begin{array}{l}\text { Taux d'implication dans la lutte } \\
\text { climatique }\end{array}$ & $\begin{array}{l}\text { "Je m' mimplique actuellement dans un projet } \\
\text { de lutte climatique. " [oui, non] }\end{array}$ \\
\hline
\end{tabular}

\section{Étude de cas - Sors de ta bulle}

L'étude de cas est une approche méthodologique empirique consistant à enquêter sur un objet spécifique afin d'en tirer une description précise qui permet de mieux comprendre un système plus large (Roy, 2010). Dans le cas présent, quatre critères justifient le recours à l'étude de cas (Gagnon, 2012). D'abord, le cadre d'évaluation de l'efficacité d'une activité d'ERCC doit être mis à l'épreuve empiriquement afin d'en examiner les forces et faiblesses potentielles. Deuxièmement, l'évaluation doit être réalisée de manière concomitante à une activité d'ERCC, dans la mesure où elle exige des mesures avant et après la tenue de l'activité. Troisièmement, le contrôle ou la manipulation des sujets d'étude - dont les participant.e.s de l'activité d'ERCC - n'est pas 
nécessaire (ni même souhaitable) pour évaluer l'efficacité de l'activité. Finalement, les bases théoriques de l'évaluation de l'efficacité des activités d'ERCC comportent encore des éléments inexpliqués - on ne sait pas, par exemple, quel modèle théorique convient le mieux - et l'étude de cas est donc utile pour explorer une nouvelle proposition de cadre d'évaluation.

17 La Fondation Monique-Fitz-Back, une organisation à but non lucratif investie dans la promotion de l'ERCC au Québec, a mis en œuvre la campagne Sors de ta bulle, visant à mobiliser les jeunes de 12 à 17 ans dans la lutte contre le changement climatique. Déclinée en plusieurs volets, la campagne comprend notamment l'organisation d'un Sommet jeunesse annuel sur le changement climatique (SJCC), une activité de deux jours réunissant quelques centaines de jeunes intéressé.e.s à en apprendre davantage sur le changement climatique et sur les différentes formes de mobilisation contre ce phénomène. Les participant.e.s sont principalement sélectionné.e.s sur la base de leur intérêt envers la problématique et de leur provenance, la Fondation Monique-Fitz-Back s'assurant d'accueillir des jeunes de toutes les régions du Québec.

L'étude de cas que nous avons menée s'inscrit dans la posture des multiples paradigmes (Teddlie et Tashakkori, 2003), soit «un modèle qui peut privilégier des données quantitatives ou qualitatives séparément, de manière séquentielle ou ensemble, de façon simultanée " à partir des méthodes qu'un.e chercheur.euse estime les plus appropriées pour atteindre l'objectif de recherche (Anadon, 2019, p.113). Plus précisément, l'étude de cas s'est ancrée dans un devis mixte séquentiel explicatif, dans lequel une première phase de collecte de données quantitatives (questionnaires en ligne) a été suivie d'une seconde phase de collecte de données qualitatives (entretien de groupe), dans le but d'explorer et d'expliquer plus en détail les résultats quantitatifs initiaux (Creswell et Plano-Clark, 2018 ; Onwuegbuzie et Leech, 2005).

Les indicateurs d'efficacité élaborés précédemment (tableau 1) ont été utilisés pour évaluer les effets de la participation des jeunes à un SJCC sur l'adoption de comportements écoresponsables individuels et collectifs.

20 La collecte de données a été effectuée avant, pendant et après le SJCC de 2019, tenu les 17 et 18 mai 2019 à Lévis, dans la région de Québec ${ }^{1}$. D’abord, deux questionnaires en ligne ont été envoyés aux 350 participant.e.s, le premier quelques jours avant l'événement et le second quelques jours après. Il était précisé qu'aucune donnée ne permettrait d'identifier les participant.e.s et que la complétion des questionnaires était facultative. Identiques et comportant des questions fermées, les questionnaires avant et après le SJCC couvraient les différents indicateurs du tableau 1: par exemple, "De façon spécifique, m'impliquer dans la lutte climatique est pour moi : (1) très important (2) assez important (3) peu important (4) pas important ». L'objectif était de comparer les résultats pour vérifier si la participation au Sommet avait eu un effet sur les différentes variables du processus d'adoption d'un comportement écoresponsable. Plus précisément, lors de l'analyse, les pourcentages des questions similaires ont été comparés entre les deux questionnaires pour mesurer cet effet.

21 Les questionnaires avant et après le SJCC ont respectivement obtenu 41 et 76 réponses (soit un taux de réponse variant entre $12 \%$ et $22 \%$ ). Il s'agit d'un échantillon de convenance, adéquat pour notre étude dans la mesure où l'objectif n'est pas de représenter avec exactitude l'ensemble des sous-catégories de participants pour en tirer des statistiques généralisables (Fortin et Gagnon, 2016). Les implications méthodologiques de ce type d'échantillonnage sont abordées dans la discussion. 

semi-dirigé de 60 minutes a été réalisé, auprès de douze participant.e.s. Provenant d'écoles et de régions variées du Québec, ces jeunes ont été sélectionné.e.s sur une base volontaire lors d'un appel à tous et à toutes en début de journée. Permettant de recueillir des données riches et complexes, l'entretien semi-dirigé était pertinent pour mieux comprendre la perspective personnelle des jeunes sur les effets de leur participation au SJCC (comme le suggèrent Creswell et Plano-Clark, 2018).

Le canevas d'entretien a été élaboré à partir de l'analyse des résultats du questionnaire « avant SJCC » et comprenait des questions ouvertes autour des trois dimensions du comportement écoresponsable (la motivation, le sentiment d'efficacité et le passage à l'action). Par exemple, à partir du constat selon lequel seulement un tiers des jeunes se considéraient déjà très renseigné.e.s sur la façon de réduire personnellement leur impact climatique, il a été pertinent d'explorer les racines de cette perception et l'effet potentiel de leur participation au SJCC. En ce sens, certaines questions ont été posées : «Vous considérez-vous plutôt démuni ou outillé pour réduire votre impact sur le climat? Pour quelles raisons? De quelle façon votre participation au SJCC a-t-elle influencé cette perception? Qu'avez-vous appris selon vous à cet égard? »

\section{Résultats}

Cette section présente d'abord un bref portrait des participant.e.s au SJCC. Elle fait ensuite état des résultats des questionnaires et de l'entretien de groupe. De façon générale, trois constats émergent en lien avec la participation des jeunes au SJCC : une augmentation de la motivation chez les jeunes, un renforcement de leur sentiment d'efficacité personnelle et une intensification de leur engagement dans la lutte climatique.

Pour la majorité des jeunes, il s'agissait d'une première participation à cet événement. Principalement engagé.e.s au $2^{\mathrm{e}}$ cycle du secondaire, les participant.e.s provenaient de 81 écoles issues de 13 régions du Québec $^{2}$. La forte majorité s'estimait déjà bien renseignée sur la crise climatique et plutôt convaincue de l'importance de s'impliquer dans la lutte, mais tout en entretenant un certain pessimisme quant à l'avenir. De plus, la majorité considérait ne pas être complètement outillée pour réduire sa propre empreinte climatique ou inciter son entourage à le faire. Néanmoins, dans son quotidien, la forte majorité se disait déjà engagée d'une manière ou d'une autre dans la lutte climatique.

\section{Augmentation de la motivation}

À la lumière de l'analyse des données, le premier constat est que la participation au SJCC a eu un effet positif sur plusieurs indicateurs de la dimension motivationnelle du processus d'adoption d'un comportement écoresponsable, et ce, à quatre égards.

D'abord, la proportion de participant.e.s estimant connaitre «très bien » les causes et les conséquences du changement climatique a augmenté (+ $37 \%)$. Lors de l'entretien de groupe, les jeunes ont expliqué que, bien que la majorité d'entre eux étaient déjà sensibilisé.e.s aux enjeux climatiques, ils et elles considéraient leurs sources actuelles 
d'information (l'école, les médias) plutôt limitées. Ils et elles estiment avoir trouvé au SJCC des sources fiables et crédibles pour mieux comprendre ce phénomène.

De plus, après le SJCC, les jeunes trouvant « très important » de jouer un rôle actif dans la société sont plus nombreux $(+20 \%)$. Plus spécifiquement, on observe une augmentation $(+21 \%)$ de la proportion des jeunes qui estiment «très important » de s'impliquer dans lutte climatique. Lors de l'entretien, plusieurs participant.e.s ont en effet affirmé ressentir un grand stress lié aux enjeux climatiques. Par exemple, une jeune a déclaré :

Dans les médias, on voit des politiciens qui ne font rien pour lutter contre le changement climatique, comme Trump, par exemple. C'est décourageant. En plus, toutes les statistiques qu'on lit montrent que la situation empire. Moi, ça me fait vraiment peur.

Pour cette raison, les jeunes étaient déjà nombreux à vouloir s'impliquer dans la lutte contre le changement climatique avant même leur participation au SJCC. Cependant, ils se sentaient parfois démunis. Dans ce contexte, les jeunes se posaient certaines questions avant d'assister au SJCC : Que faut-il faire exactement? Cela est-il suffisant pour inverser la tendance actuelle ? À cet égard, les jeunes ont affirmé y avoir trouvé une forme de soulagement qui a contribué à nourrir leur motivation: "Ce qui est apaisant, c'est que ce sont des experts du milieu qui viennent nous rassurer, [...] [nous dire] que c'est encore possible de changer les choses si on se mobilise ", a expliqué un participant.

Finalement, les résultats montrent un renforcement de la perception concernant l'implication des jeunes dans la lutte climatique (taux d'accord passant de 7,3 / 10 à $8,6 / 10)$. Lors de l'entretien, les participant.e.s ont rapporté que le grand nombre de jeunes présents à l'évènement avait chassé leur sentiment d'isolement : «Je n'avais pas réalisé à quel point il y a des jeunes qui s'activent au Québec; ça me motive, parce que j'avais parfois l'impression d'être seul dans mon coin ", a déclaré l'un des participant.s.

31 Toutefois, la participation au SJCC n'a pas eu d'impact important (+3\%) sur la perception de l'utilité des actions individuelles pour lutter contre le changement climatique. Pour les participant.e.s à l'entretien de groupe, cela semblait être un fait acquis.

32 Dans le même ordre d'idées, le SJCC n'a pas eu d'effet significatif sur la perception des jeunes selon laquelle leur implication dans la lutte climatique est généralement perçue de manière positive par leur entourage (taux d'accord passant de 8,1 à 8,4/10). Autrement dit, les jeunes bénéficiaient déjà d'une forme d'approbation sociale de la part de leurs pairs (famille, amis, etc.). Toutefois, plusieurs participant.e.s ont affirmé ressentir une forme de pression sociale les incitant à maintenir un engagement environnemental parfait en tout temps. Un jeune a déclaré :

On est tellement rendus dans une société de performance, à l'école, dans les sports, partout, que c'est rendu la même chose dans ton implication. Il faut que tu sois un végétarien parfait ou que tu élimines complètement le plastique de ta vie, sinon tu te le fais reprocher.

33 En effet, plusieurs jeunes ont affirmé se sentir scruté.e.s par leurs pairs, au point où certain.e.s ont choisi de vivre leur mode de vie écoresponsable dans l'ombre. Certains jeunes ont déclaré qu'en participant au SJCC, ils et elles ont réalisé qu'un mode de vie parfait n'est pas nécessaire pour réduire leur empreinte climatique, ce qui a contribué à abaisser la pression sociale ressentie. 

changement climatique après le SJCC. En effet, bien que la participation au SJCC n'ait pas influencé la proportion de jeunes affirmant s'impliquer dans un projet de lutte climatique ( $71 \%$ avant et après le SJCC), ils et elles sont désormais plus nombreux et nombreuses $(+18 \%)$ à se décrire comme «très actifs » et "très actives » dans cette lutte. Selon les participant.e.s à l'entretien, l'acquisition de compétences communicationnelles, la création d'un réseau de pairs et la découverte de nouvelles idées de projets sont des outils supplémentaires permettant aux jeunes d'accroitre leur engagement: "Le fait que ce soit à l'échelle provinciale a vraiment un impact, parce qu'on s'ajoute sur les réseaux sociaux; si j'implante un nouveau projet, je sais que je 
peux écrire à tout le monde pour avoir des conseils; le Sommet crée des liens ", a indiqué une participante.

41 De plus, certains jeunes ont expliqué leur perception de l'intensification de leur engagement par la prise de conscience de l'étendue de leur champ d'action, auparavant restreint à leur milieu scolaire. En participant au SJCC, les jeunes ont constaté que la mobilisation climatique pouvait se faire à plusieurs échelles: politique, communautaire, familiale, individuelle, etc. Par exemple, une participante a rapporté avoir appris qu'« on peut écrire aux ministres, écrire aux députés, etc.; ce sont des choses simples, mais qui peuvent vraiment faire une différence.»

\section{Au bilan}

Le tableau 2 expose les réponses comparées au questionnaire avant et après le SJCC au regard de la motivation chez les jeunes, du renforcement de leur sentiment d'efficacité personnelle et du passage à l'action, se traduisant par une intensification de leur engagement dans la lutte climatique.

Tableau 2. Résultats comparés des indicateurs quantitatifs avant et après le SJCC 2019

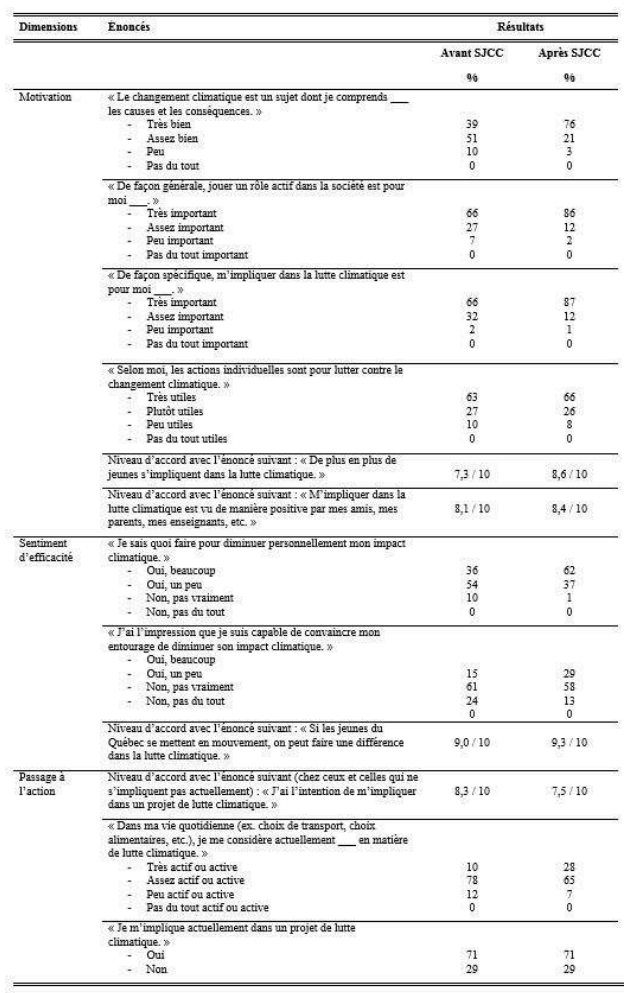

\section{Discussion}

Pour les praticien.ne.s de l'ERCC en milieu non formel, l'évaluation de l'efficacité de leurs activités constitue un défi, notamment en raison de l'absence de méthode permettant d'observer l'ensemble du processus d'adoption d'un comportement écoresponsable à l'échelle individuelle ou collective (Guevara et coll., 2009; Riemer et coll., 2014). 
En proposant une liste d'indicateurs s'appuyant sur un modèle théorique existant (Klöckner, 2015), notre recherche répond à cette préoccupation. Son principal intérêt est de mettre en lumière les effets d'une activité d'ERCC sur les différentes variables du processus d'adoption comportementale.

De plus, ce cadre offre une souplesse méthodologique aux praticien.ne.s puisqu'il sied autant aux méthodes quantitatives que qualitatives, au sein d'un devis mixte. Il peut donc s'intégrer aux activités d'évaluation les mieux adaptées au contexte des praticien.ne.s, selon les ressources (financières, temporelles, logistiques, etc.) dont ils et elles disposent.

Une limite importante concerne toutefois le fait que les résultats reposent sur des déclarations autorapportées par les jeunes. Conséquemment, il existe le risque d'un biais de désirabilité sociale, où une personne pourrait être tentée d'offrir la réponse qu'elle estime être en accord avec la norme sociale. Par exemple, un.e jeune pourrait croire qu'il est très mal vu de ne pas vouloir jouer un rôle actif dans la société ou de ne pas être préoccupé par la crise climatique. Il importe cependant de rappeler que les normes sociales varient d'un segment de population à l'autre et, pour cette raison, les énoncés proposés dans le cadre d'évaluation pourraient tout autant commander des réponses positives que négatives, selon la norme sociale dominante du jeune interrogé. Sans pouvoir éliminer cette limite, une façon de mieux l'appréhender consiste à bien identifier les normes sociales du groupe étudié, notamment par le biais d'une méthode qualitative, puis à valider si les répondant.e.s ressentent, de façon générale, une forte pression à se conformer aux normes du groupe. C'est notamment ce qui a été fait dans le cadre de l'étude de cas du Sommet jeunesse sur le changement climatique.

7 Ultimement, l'application au cas du Sommet jeunesse sur le changement climatique, déployé au sein de la campagne Sors de ta bulle de la Fondation Monique-Fitz-Back, suggère que l'utilisation d'un devis mixte peut permettre non seulement de rendre compte de l'efficacité de l'activité à stimuler l'adoption de comportements écoresponsables auprès des participant.e.s, mais également d'apporter des éléments d'explication à cette influence. En croisant deux méthodes de collecte de données (questionnaires et entretien de groupe), le devis mixte visait à réduire les faiblesses d'une méthode par les forces d'une autre (Karsenti et Demers, 2018). Ici, la méthode quantitative utilisée seule n'aurait pas permis de comprendre pourquoi, précisément, la participation au SJCC a provoqué les effets observés. De manière complémentaire, la méthode qualitative a compensé cette faiblesse en fournissant ces explications.

Par exemple, la comparaison des réponses au questionnaire avant et après le SJCC a montré que les jeunes ont développé une motivation accrue à s'impliquer dans la lutte climatique. Or, l'entretien de groupe a mis en exergue que ce gain de motivation était notamment dû à un apaisement de l'écoanxiété vécue par de nombreux.ses participant.e.s, ce que le questionnaire seul n'aurait pas permis d'identifier. En effet, l'écoanxiété se définit comme une forme de détresse causée par les changements environnementaux en général, provoquant une diversité d'émotions comme la peur, la colère, le sentiment d'impuissance et la fatigue (Albrecht, 2011). Plusieurs participant.e.s ont déclaré vivre de telles émotions. La présence d'expert.e.s au SJCC affirmant qu'il n'est pas trop tard pour limiter les conséquences du changement climatique a contribué à calmer l'angoisse des participant.e.s. Comprendre cette réalité par le biais de l'entretien de groupe a ainsi permis d'expliquer une partie de l'efficacité de l'activité du Sommet dans le cadre de la campagne Sors de ta bulle. 

quoi le fait de rencontrer des centaines de jeunes impliqué.e.s dans la lutte climatique a contribué à stimuler leur motivation, et ce, pour trois raisons. D'une part, bien que la majorité des participant.e.s étaient déjà engagé.e.s dans la lutte climatique, plusieurs ont rapporté se sentir isolé.e.s dans leurs communautés respectives. Par exemple, les comités environnementaux locaux ne comptent parfois que quelques membres, ce qui peut alimenter des sentiments d'isolement et d'impuissance, soit d'importants freins à l'action. En effet, Cojuharenco et coll. (2016) rapportent que ces émotions expliquent souvent la baisse d'intérêt envers l'implication sociale ou environnementale. Ainsi, faire partie d'un grand rassemblement de jeunes motivés a contribué à diminuer ces sentiments négatifs. D'autre part, cela a nourri l'espoir chez les participant.e.s, une émotion positive stimulant l'engagement des jeunes dans la lutte climatique (Ojala, 2012). Finalement, en émergeant de leur cercle social immédiat, les participant.e.s ont pu tisser des liens avec d'autres jeunes partageant leurs convictions environnementales. Cela constitue un nouveau groupe social de référence auquel les jeunes peuvent désormais s'identifier et dont les normes pourront exercer une forte influence, parfois même plus forte que celle de leur entourage (Hogg, 2003; Keizer et Schultz, 2013). En bref, ces exemples montrent la complémentarité et la richesse des données obtenues par le biais de l'intégration du cadre d'évaluation dans un devis méthodologique mixte.

50 Dans le cas de l'évaluation de la campagne Sors de ta bulle, plus spécifiquement de l'activité du SJCC, deux limites méthodologiques principales méritent d'être rappelées. D'abord, les taux de réponse inégaux entre les questionnaires «avant » et «après SJCC » ne permettent pas d'établir des comparaisons statistiques robustes. Par exemple, on ne peut pas garantir que ce sont exactement les mêmes participant.e.s qui ont répondu aux questionnaires. Par ailleurs, l'entretien de groupe a été tenu à la fin de l'événement, témoignant ainsi d'effets à très court terme. Il aurait été intéressant de réaliser d'autres activités d'évaluation quelques semaines - voire quelque mois - après le SJCC pour vérifier si les effets observés ont perduré.

\section{Conclusion}

51 Le rôle joué par l'ERCC dans la lutte contre le changement climatique, tant dans les milieux d'enseignement formels que non formels, est de plus en plus reconnu au Québec, comme en témoigne la proposition de Stratégie québécoise d'éducation en matière d'environnement et d'écocitoyenneté (Centr'ERE, 2019). Dans ce contexte il importe de développer des outils permettant d'évaluer l'efficacité de ces activités sur le terrain. Or, nous avons vu que la documentation scientifique consultée indique que ces outils d'évaluation sont, pour l'instant, peu nombreux et souffrent souvent de l'absence d'assises théoriques solides.

Cet article constitue ainsi une contribution à la réflexion autour de cet enjeu pratique, en proposant un cadre d'évaluation s'enracinant dans un modèle théorique de l'adoption d'un comportement écoresponsable. Il est souhaité que d'autres praticiens.nes du milieu non formel utilisent ce cadre d'évaluation dans différents contextes, afin d'en relever les forces et, potentiellement, quelques limites qui pourraient être corrigées dans une version revisitée.

Éducation relative à l'environnement, Volume 17-1 | 2022 
Appliqué au cas de la campagne Sors de ta bulle de la Fondation Monique-Fitz-Back, ce cadre a permis de démontrer qu'une activité d'ERCC comme le Sommet jeunesse annuel sur le changement climatique peut avoir des effets mesurables, à court terme du moins, sur la motivation des jeunes, leur sentiment d'efficacité et leur engagement dans la lutte climatique. Une étude longitudinale et des ressources financières importantes permettraient de vérifier la persistance de ces effets à long terme.

\section{BIBLIOGRAPHIE}

Albrecht G. (2011) Chronic environmental change : Emerging 'psychoterratic' syndromes. Dans I. Weissbecker (Dir.), Climate Change and Human Well-Being. International and Cultural Psychology (p. 43-56). New York, NY : Springer.

Alisat, S. et Riemer, M. (2015). The environmental action scale : Development and psychometric evaluation. Journal of Environmental Psychology, 43, 13-23.

Anadón, M. (2019). Les méthodes mixtes : implications pour la recherche « dite » qualitative. Recherches qualitatives, 38 (1), 105-123.

Arnett, J.J. (2006). Emerging adulthood : Understanding the new way of coming of age. Dans J.J. Arnett et J.L. Tanner (Dir.), Emerging Adults in America : Coming of Age in the 21st Century. (p. 3-19). Washington, DC : American Psychological Association.

Arnold, H.E., Cohen, F.G., et Warner, A. (2009). Youth and environmental action : Perspectives of young environmental leaders on their formative influences. Journal of Environmental Education, 40(3), 27-36.

Ballantyne, R., Connell, S. et Fien, J. (1998). Students as catalysts of environmental change : A framework for researching intergenerational influence through environmental education. Environmental Education Research, 4(3), 285-298.

Bamberg, S. (2013). Processes of change. Dans L. Steg, A. Van Den Berg, et J. De Groot (Dir.), Environmental Psychology : An Introduction (p. 267-279). Chichester : BPS Blackwell.

Bamberg, S. et Möser, G. (2007). Twenty years after Hines, Hungerford, and Tomera : A new metaanalysis of psycho-social determinants of pro-environmental behaviour. Journal of Environmental Psychology, 27(1), 14-25.

Beaud, J.-P. (2010). L'échantillonnage. Dans B. Gauthier (Dir.), Recherche sociale : de la problématique à la collecte de données (5e éd., p. 251-284). Québec : Presses de l'Université du Québec.

Blythe, C. et Harré, N. (2012). Inspiring youth sustainability leadership : Six elements of a transformative youth eco-retreat. Ecopsychology, 4(4), 336-344.

Bonnett, M. (2002). Education for sustainability as a frame of mind. Environmental Education Research, 8(1), 9-20

Centre de recherche en éducation et formation relatives à l'environnement et à l'écocitoyenneté (Centr'ERE) (2019). Stratégie québécoise d'éducation en matière d'environnement et d'écocitoyenneté. Montréal : Université du Québec à Montréal. Récupéré de https://www.coalition-education- 
environnement-ecocitoyennete.org/wp-content/uploads/2019/07/Strategie-Editioncomplete.pdf

Champagne St-Arnaud, V. et Daignault, P. (2020). Baromètre de l'action climatique 2020 : Disposition des Québécois et des Québécoises face aux défis climatiques. Québec : Université Laval. Récupéré de https://unpointcinq.ca/barometre-de-laction-climatique-2020

Champagne-Poirier, O., Samson, G., Chastenay, P. et Luckerhoff, J. (2017). Les musées de sciences et de technologie l'éducation formelle et l'éducation non formelle. Dans M.-C. Larouche, J. Luckerhoff et S. Labbé (dir.), Regards interdisciplinaires sur les publics de la culture (p. 63-80). Québec : Presses de l'Université du Québec.

Cojuharenco, I., Cornelissen, G. et Karelaia, N. (2016). Yes, I can : Feeling connected to others increases perceived effectiveness and socially responsible behavior. Journal of Environmental Psychology, 48, 75-86.

Creswell, J.W. et Plano Clark, V.L. (2018). Designing and Conducting Mixed Methods Research (3 ${ }^{\mathrm{e}}$ éd.). Thousand Oaks : SAGE.

Davis, J. M. (2010). What is early childhood education for sustainbility? Dans J. M. Davis (Dir.), Young Children and the Environment : Early Education for Sustainability (p. 21-42). Melbourne : Cambridge University Press.

Dittmer, L., Mugagga, F., Metternich, A., Schweizer-Ries, P., Asiimwe, G. et Riemer, M. (2018). “We can keep the fire burning" : building action competence through environmental justice education in Uganda and Germany. Local Environment, 23(2), 144-157.

Fortin, M.-F. et Gagnon, J. (2016). Fondements et étapes du processus de recherche : Méthodes quantitatives et qualitatives ( $3^{\mathrm{e}}$ édition). Montréal, Chenelière education.

Fishbein, M. et Ajzen, I. (2010). Predicting and Changing Behavior : The Reasoned Action Approach. New York : Psychology Press.

Gagnon, Y.-C. (2012). L'étude de cas comme méthode de recherche (2e éd). Sainte-Foy, Presses de l'Université du Québec.

Goldberg, M., Gustafson, A., Rosenthal, S., Kotcher, J., Maibach, E., et Leiserowitz, A. (2020). For the first time, the Alarmed are now the largest of Global Warming's Six Americas. Yale University and George Mason University. New Haven, CT : Yale Program on Climate Change Communication.

Groupe d'experts intergouvernemental sur l'évolution du climat (2018). Summary for Policymakers. Dans V. Masson-Delmotte, P. Zhai, H.-O. Pörtner, D. Roberts, J. Skea, P.R. Shukla, A. Pirani, W. Moufouma-Okia, C. Péan, R. Pidcock, S. Connors, J.B.R. Matthews, Y. Chen, X. Zhou, M.I. Gomis, E. Lonnoy, T. Maycock, M. Tignor et T. Waterfield (Dir.), Global Warming of $1.5^{\circ} \mathrm{C}$. An IPCC Special Report on the impacts of global warming of $1.5^{\circ} \mathrm{C}$ above pre-industrial levels and related global greenhouse gas emission pathways, in the context of strengthening the global response to the threat of climate change, sustainable development, and efforts to eradicate poverty. Genève : Groupe d'experts intergouvernemental sur l'évolution du climat. Récupéré de https://www.ipcc.ch/2018/10/08/ summary-for-policymakers-of-ipcc-special-report-on-global-warming-of-1-5c-approved-bygovernments/

Ginwright, S., et James, T. (2002). From assets to agents of change : social justice, organizing, and youth development. New Directions for Youth Development, 2002(96), 27-46.

Guevara, R., Whelan, J. et Flowers, R. (2009). Popular and informal environmental education : the need for more research in an « emerging » field of practice. REPORT - Zeitschrift Für Weiterbildungsforschung, 32(2), 35-50. 
Hogg, M.A. (2003). Social identity. Dans M.R. Leary et J.P. Tangney (Dir.), Handbook of self and identity (p. 462-479). New York : Guilford Press.

Karsenti, T. et Demers, S. (2018). L'étude de cas. Dans T. Karsenti et L. Savoie-Zajc (dir.), La recherche en éducation : étapes et approches (4éd.) (p. 289-316). Montréal : Presses de l'Université de Montréal.

Keizer, K., et Schultz, P.W. (2013). Social norms and pro-environmental behaviour. Dans L. Steg, A. Van den Berg et J.I.M. de Groot (Dir.), Environmental psychology : An introduction (p. 153-163). Chichester : BPS Blackwell.

Kerr, N. L. (1989). Illusions of efficacy : The effects of group size on perceived efficacy in social dilemmas. Journal of Experimental Social Psychology, 25(4), 287-313.

Kerry, J., Pruneau, D., Cousineau, M., Mallet, M.-A., Laliberté, B. et Langis, J. (2014). Faire naître l'espoir et l'auto-efficacité chez les jeunes par l'action environnementale communautaire. Canadian Journal of Education/Revue Canadienne De l'éducation, 36(4), 3-32.

Klöckner, C.A. (2015). The Psychology of Pro-environmental Communication : Beyond Standard Information Strategies. Houndmills : Palgrave Macmillan.

Klöckner, C.A. et Oppedal, I.O. (2011). General vs. domain specific recycling behaviour - Applying a multilevel comprehensive action determination model to recycling in Norwegian student homes. Resources, Conservation and Recycling, 55(4), 463-471.

Jensen, B.B. (2002). Knowledge, action and pro-environmental behaviour. Environmental Education Research, 8(3), 325-334.

Jensen, B. B., et Schnack, K. (1997). The action competence approach in environmental education. Environmental Education Research, 3(2), 163-178.

Joanes, T., Gwozdz, W., et Klöckner, C.A. (2020). Reducing personal clothing consumption : A cross-cultural validation of the comprehensive action determination model. Journal of Environmental Psychology.

Ojala, M. (2012). Hope and climate change : The importance of hope for environmental engagement among young people. Environmental Education Research, 18(5), 625-642.

Onwuegbuzie, A. J., et Leech, N. L. (2005). Taking the "q" out of research : Teaching research methodology courses without the divide between quantitative and qualitative paradigms. Quality and Quantity, 39(3), 267-296.

Riemer, M., Lynes, J. et Hickman, G. (2014). A model for developing and assessing youth-based environmental engagement programmes. Environmental Education Research, 20(4), 552-574.

Riemer, M., Voorhees, C., Dittmer, L., Alisat, S., Alam, N., Sayal, R., Bidisha, S.H., De Souza, A., Lynes, J., Metternich, A., Mugagga, F. et Schweizer-Ries, P. (2016). The youth leading environmental change project : A mixed-method longitudinal study across six countries. Ecopsychology, 8(3), 174-187.

Roy, S.N. (2010). L'étude de cas. Dans B. Gauthier (Dir.), Recherche sociale : de la problématique à la collecte de données (5éd., p. 199-226). Québec : Presses de l'Université du Québec.

Sauvé, L. (2013). Au cœur des questions socio-écologiques : des savoirs à construire, des compétences à développer. Éducation relative à l'environnement - Regards, Recherches, Réflexions, 11, 19-40. Récupéré de https://journals.openedition.org/ere/662 
Schusler, T.M., Krasny, M.E., Peters, S.J. et Decker, D.J. (2009). Developing citizens and communities through youth environmental action. Environmental Education Research, 15(1), 111-127.

Sopha, B.M. et Klöckner, C.A. (2011). Psychological factors in the diffusion of sustainable technology : A study of Norwegian households' adoption of wood pellet heating. Renewable and Sustainable Energy Reviews, 15(6), 2756-2765.

Simard, C. et Samson, G. (2018, mars). Activités en collaboration avec le milieu éducatif non formel et construction d'un rapport aux vivants d'élèves de 12 à 15 ans. Communication présentée au symposium «Construction d'un rapport aux vivants en milieu scolaire » lors de la $10^{\mathrm{e}}$ rencontre de l'Association pour la recherche en didactique des sciences et des technologies (ARDIST). SaintMalo, France.

Simard, C. et Samson, G. (soumis). Teaching with local biodiversity : Exploratory study on the contributions of interventions for students aged 12 to 14, within the cognitive, affective and behavioural dimensions. Canadian Journal of Education.

Steg, L., et Vlek, C. (2009). Encouraging pro-environmental behaviour : An integrative review and research agenda. Journal of Environmental Psychology, 29(3), 309-317.

Stern, P.C. (2000). Toward a coherent theory of environmentally significant behavior. Journal of Social Issues, 56(3), 407-424.

Stocknes, P.E. (2015). What We Think About When We Try Not to Think About Global Warming. White River Junction : Chelsea Green Publishing.

van den Broek, K.L., Walker, I. et Klöckner, C.A. (2019). Drivers of energy saving behaviour : The relative influence of intentional, normative, situational and habitual processes. Energy Policy, 132, 811-819.

Teddlie, C. et Tashakkori, A. (2003). Major issues and controversies in the use of mixed methods in the social and behavioral sciences. Dans A. Tashakkori, et C. Teddlie (Éds), Handbook of mixed methods in social and behavioral research (pp. 3-50). Thousand Oaks, CA : Sage.

Trott, C.D., Rockett, M.L., Grav, E.-S., Lam, S., Even, T.L. et Frame, S.M. (2020). “Another Haiti starting from the youth" : Integrating the arts and sciences for empowering youth climate justice action in Jacmel, Haiti. Community Psychology in Global Perspective, 6(2), 48-70

\section{NOTES}

1. L'évaluation a été effectuée à deux reprises lors des éditions de 2018 et de 2019 des SJCC, mais pour les fins de la présente étude de cas, seuls la démarche et les résultats de l'évaluation de 2019 sont présentés.

2. Ces données ont été fournies par la Fondation Monique-Fitz-Back. 


\section{RÉSUMÉS}

Les outils permettant d'évaluer l'efficacité de l'éducation relative au changement climatique en milieu non formel sont peu nombreux et souffrent souvent de l'absence d'assises théoriques solides. Dans le contexte de la campagne Sors de ta bulle menée par la Fondation Monique-FitzBack, un cadre d'évaluation novateur reposant sur douze indicateurs a été élaboré. Ces indicateurs ont servi à évaluer les effets de la participation de jeunes de 12 à 17 ans au Sommet jeunesse sur le changement climatique, l'une des activités de la campagne. Un devis mixte combinant la réalisation d'une enquête en ligne auprès des participant.e.s et un entretien de groupe avec douze d'entre eux a permis de constater que la campagne Sors de ta bulle a contribué à stimuler efficacement le processus d'adoption de comportements écoresponsables selon trois axes: augmentation de la motivation, renforcement du sentiment d'efficacité personnelle et intensification de l'engagement dans la lutte climatique.

There are few tools to assess the effectiveness of climate change education in non-formal context. Moreover, they often lack solid theoretical foundations. In the context of the Sors de ta bulle campaign led by the Fondation Monique-Fitz-Back, an innovative evaluation framework based on twelve indicators was developed. These indicators were used to assess the impacts of the participation of high school students in the Youth Climate Change Summit, one of the campaign activities. A mixed-methods design combining an online survey and a group interview showed that the impacts on participants were threefold : increasing motivation, strengthening of perceived personal efficacy and intensification of the engagement in the fight against climate change.

\section{INDEX}

Keywords : climate change education, non-formal context, high school students, evaluation framework, proenvironmental behavior, environmental psychology.

Mots-clés : éducation relative au changement climatique, milieu non formel, école secondaire, cadre d'évaluation, comportement écoresponsable, psychologie environnementale

Index géographique : Québec

\section{AUTEURS}

\section{VALÉRIANE CHAMPAGNE ST-ARNAUD}

Professeure au Département de marketing de l'Université Laval, Valériane Champagne St-Arnaud est spécialiste de la communication environnementale et du marketing social appliqué aux enjeux de comportements écoresponsables. Ses travaux de recherche portent sur l'évaluation de l'efficacité du marketing social et le transfert de connaissances entre chercheur.e.s et praticien.ne.s du marketing.

\section{GHISLAIN SAMSON}

Professeur au Départements des sciences de l'éducation de l'Université du Québec à TroisRivières (UQTR), Ghislain Samson est actuellement doyen de la gestion académique des affaires professorales à cette même université. Spécialiste de la didactique des sciences et du transfert des apprentissages en mathématiques et en sciences, il s'intéresse également aux questions 
relatives au curriculum scolaire, à l'interdisciplinarité et à l'éducation relative à l'environnement. 